\title{
Xclaim: a graphical interface for the calculation of core-hole spectroscopies
}

\author{
Javier Fernández-Rodríguez, ${ }^{1,2}$ Brian Toby, ${ }^{2}$ and Michel van Veenendaal ${ }^{1,2}$ \\ ${ }^{1}$ Department of Physics, Northern Illinois University, DeKalb, Illinois 60115, USA \\ ${ }^{2}$ Advanced Photon Source, Argonne National Laboratory, \\ 9700 South Cass Avenue, Argonne, Illinois 60439, USA
}

(Dated: March 31, 2015)

\begin{abstract}
Xclaim (x-ray core level atomic multiplets) is a graphical interface for the calculation of corehole spectroscopy and ground state properties within a charge-transfer multiplet model taking into account a many-body Hamiltonian with Coulomb, spin-orbit, crystal-field, and hybridization interactions. Using Coulomb and spin-orbit parameters calculated in the Hartree-Fock limit and ligand field parameters (crystal-field, hybridization and charge-transfer energy) the program calculates x-ray absorption spectroscopy (XAS), x-ray photoemission spectroscopy (XPS), photoemission spectroscopy (PES) and inverse photoemission (IPES). The program runs on Linux, Windows and MacOS platforms.
\end{abstract}

Keywords: X-ray Absorption Spectroscopy,X-ray Photoemission,Crystal Field,Strongly correlated Materials

\section{INTRODUCTION}

Multiplet ligand-field theory (MLFT) or small cluster calculations [1-4] are useful approaches for calculating xray spectroscopy on strongly correlated materials where the spectral lineshape is dominated by strong multiplet effects arising from the Coulomb interactions between the valence electrons and between the valence electrons and the core hole. Since the eigenfunction of a Coulomb multiplet often involves several Slater determinants, they are often poorly described by effective single-particle models, such as density functional theory. For MLFT, one considers a single ion and the effects of the ligands are described by an effective crystal field. This approach often works well when describing x-ray absorption spectroscopy. For $\mathrm{x}$-ray photoemission, screening effects are stronger and the ligands have to be included explicitly. This is generally known as small-cluster calculations. The spectra are calculated by constructing a many-body Hamiltonian for the system using full configuration-interaction, i.e. taking into account in the basis states any possible combination of Slater determinants and has the advantage of accurately treating the Coulomb interaction in the metal ion. This approach has been used with great success to describe x-ray spectra. [5-15].

In this paper we discuss the calculation of core-hole spectroscopy in terms of a multiplet Hamiltonian with the model implemented in Xclaim [16]. Xclaim is a code to calculate different types of X-ray spectra within the ionic or small-cluster limit. The program allows flexible input and output via a graphical user interface. The paper is outlined as follows. First, we give an overview of the model Hamiltonian used in the calculation of the spectra. We discuss the different interactions included in the many-body Hamiltonian. Subsequently, we describe the various spectroscopies that can be calculated: $\mathrm{x}$ ray absorption spectroscopy (XAS), x-ray photoemission spectroscopy (XPS), photoemission spectroscopy (PES) and inverse photoemission (IPES). The final Section contains a description of the graphical interface for the cal- culation of spectra and ground state properties.

\section{MODEL HAMILTONIAN}

The Hamiltonian for MLFT and small-cluster calculations, can be split into the following terms

$$
H=H_{\text {atomic }}+H_{\mathrm{CF}}+H_{\text {hybridization }},
$$

where $H_{\text {atomic }}$ describes the central ion where the x-ray transition takes place. The last two terms on the righthand side describe the effects of the surrounding ions: $H_{\mathrm{CF}}$ describes the effects of the ligands as an effective point-charge crystal field; $H_{\text {hybridization }}$ is included for small-cluster calculations and describes the hybridization of the central ion with the nearest-neightbor ligand ions.

The Hamiltonian for the electrons on the central ion is given by

$$
\begin{aligned}
H_{\text {atomic }}= & \sum_{i} \frac{\mathbf{p}_{i}^{2}}{2 m}-\sum_{i} \frac{Z e^{2}}{r_{i}}+\sum_{i<j} \frac{e^{2}}{\left|\mathbf{r}_{i}-\mathbf{r}_{j}\right|} \\
& +\sum_{i} \zeta\left(r_{i}\right) \mathbf{l}_{i} \cdot \mathbf{s}_{i}-\boldsymbol{\mu} \cdot \mathbf{B},
\end{aligned}
$$

where the indices $i$ and $j$ run over all electrons of the ion. The first term in $H_{\text {atomic }}$ is the kinetic energy, where $\mathbf{p}_{i}$ and $m$ are the momentum and the mass of the electrons, respectively. The second term on the right-hand side is the potential energy of the nucleus, where $Z$ is the atomic number. These two terms lead to the binding energy for the electrons. The next term is the Coulomb interaction between the electrons. The Coulomb interactions that include electrons in a closed shell lead to an effective change in the binding energy. The interaction between two electrons that are both in open shells leads to multiplet structure that can often be clearly observed in the spectral line shape. The fourth term is the spin-orbit interaction, where $\zeta\left(r_{i}\right)$ is the radial part of the spin-orbit interaction. The last term is an external magnetic field $\mathbf{B}$ 
with $\boldsymbol{\mu}$ is the total magnetic moment $\boldsymbol{\mu}=-\mu_{B}\left(\mathbf{L}+g_{S} \mathbf{S}\right)$ with $g_{S} \approx 2$ the spin gyromagnetic ratio. The interaction is weak but plays a crucial role in lifting the degeneracy of the ground state for magnetic systems. Let us consider the interactions in $H$ in more detail.

\section{A. Coulomb and spin-orbit interactions}

In the evaluation of the matrix elements of the Hamiltonian, the angular part, resulting from the integrals over the spherical harmonics of the atomic wavefunctions, can be expressed in terms of Clebsch-Gordan coefficients or $3 j$ symbols. The radial parts of the matrix elements involve integrals over the radial atomic wavefunctions and therefore explicitly depend on the effective central-field potential that an electron in a particular orbital experiences. Whereas the angular matrix elements can be evaluated analytically, the radial matrix elements need to be calculated numerically. In the calculations, the Hartree-Fock self-consistent atomic field for an isolated ion, as implemented in Cowan's atomic multiplet program RCN $[17,18]$, is used. The resulting radial wavefunctions $P_{n l}(r)$, where $n$ is the principal quantum number and $l$ is the angular momentum quantum number, can be used to evaluate the matrix elements.

For the spin-orbit interaction this gives

$$
\zeta_{n l}=\frac{\alpha^{2}}{2} \int_{0}^{\infty} \frac{1}{r}\left(\frac{d U_{n l}}{d r}\right)\left|P_{n l}(r)\right|^{2} r^{2} d r,
$$

$\alpha$ is the fine structure constant. In the matrix element the radial part of the interaction $\zeta\left(r_{i}\right)$ from Eq. (2) can be expressed in terms of the derivative of the effective central-field potential energy $U_{n l}$. The spin-orbit interaction mixes the orbital and spin quantum numbers. This can change the ground-state symmetry, which can significantly alter the spectral line shape. In the final state, the spin-orbit of a core-shell with $l_{c}>0$ is often large enough to separate the spectrum into two distinct edges, for example the $L_{2}\left(2 p_{1 / 2}\right)$ and $L_{3}\left(2 p_{3 / 2}\right)$ edges for transitionmetal compounds.

For the electron-electron interaction, we can make a multipole expansion of $1 / r_{i j}$ with $r_{i j}=\left|\mathbf{r}_{i}-\mathbf{r}_{j}\right|$

$$
\frac{e^{2}}{\left|\mathbf{r}_{i}-\mathbf{r}_{j}\right|}=e^{2} \sum_{k} \frac{r_{<}^{k}}{r_{>}^{k+1}} \mathbf{C}^{k}\left(\hat{\mathbf{r}}_{i}\right) \cdot \mathbf{C}^{k}\left(\hat{\mathbf{r}}_{j}\right),
$$

where $r_{<,>}$is the lesser/greater of $r_{i}$ and $r_{j} ; \mathbf{C}^{k}$ is a tensor of renormalized spherical harmonics whose components are related to the spherical harmonics $C_{q}^{k}=$ $\sqrt{\frac{4 \pi}{2 k+1}} Y_{k q} ; \hat{\mathbf{r}}=\mathbf{r} / r$ is a shorthand for the angular coordinates $\theta$ and $\varphi$ in spherical polar coordinates. The Coulomb interaction is customarily parametrized in terms of the radial integrals

$$
\begin{gathered}
R_{n_{1} l_{1} n_{2} l_{2} n_{3} l_{3} n_{4} l_{4}}^{k}=e^{2} \int_{0}^{\infty} d r_{1} r_{1}^{2} \int_{0}^{\infty} d r_{2} r_{2}^{2} \frac{2 r_{<}^{k}}{r_{>}^{k+1}} \\
P_{n_{4} l_{4}}\left(r_{1}\right) P_{n_{3} l_{3}}\left(r_{2}\right) P_{n_{2} l_{2}}\left(r_{2}\right) P_{n_{1} l_{1}}\left(r_{1}\right)
\end{gathered}
$$

For two atomic orbitals, the integrals are divided into direct $F_{n l ; n^{\prime} l^{\prime}}^{k}=R_{n l n^{\prime} l^{\prime} n^{\prime} l^{\prime} n l}^{k}$ and exchange $G_{n l ; n^{\prime} l^{\prime}}^{k}=$ $R_{n l n^{\prime} l^{\prime} n l n^{\prime} l^{\prime}}$ parts. In addition, one has $F_{n l ; n l}^{k}=G_{n l ; n l}^{k}$. The matrix element for the Coulomb interaction between two electrons with orbital angular momentum $l$ and $l^{\prime}$ can be written in

$$
\begin{aligned}
& \left\langle n l m_{4} \sigma ; n^{\prime} l^{\prime} m_{3} \sigma^{\prime}|H| n^{\prime} l^{\prime} m_{2} \sigma^{\prime} ; n l m_{1} \sigma\right\rangle= \\
& \quad\left[\sum_{k q} F_{n l ; n^{\prime} l^{\prime}}^{k}\left\langle l m_{4}\left|C_{q}^{k}\right| l m_{1}\right\rangle\left\langle l^{\prime} m_{3}\left|\left[C_{q}^{k}\right]^{*}\right| l^{\prime} m_{2}\right\rangle\right. \\
& \left.\quad-\delta_{\sigma \sigma^{\prime}} G_{n l ; n^{\prime} l^{\prime}}^{k}\left\langle l^{\prime} m_{3}\left|C_{q}^{k}\right| l m_{1}\right\rangle\left\langle l m_{4}\left|\left[C_{q}^{k}\right]^{*}\right| l m_{2}\right\rangle\right]
\end{aligned}
$$

where the matrix elements of the normalized spherical harmonics $C_{q}^{k}$ are given by

$$
\left\langle l^{\prime} m^{\prime}\left|C_{q}^{k}\right| l m\right\rangle=(-1)^{m}\left[l l^{\prime}\right]^{1 / 2}\left(\begin{array}{lll}
l^{\prime} & k & l \\
0 & 0 & 0
\end{array}\right)\left(\begin{array}{ccc}
l^{\prime} & k & l \\
-m^{\prime} & q & m
\end{array}\right)
$$

and $\left[l l^{\prime}\right]$ denotes $(2 l+1)\left(2 l^{\prime}+1\right)$.

For ionic materials, the Hartree-Fock values of $F^{k}$ and $G^{k}$ are reduced customarily to $80 \%$ to account for the intraatomic configuration-interaction effects. Reductions to less than $80 \%$ can be used to mimic the effect of hybridization in a simpler crystal field model without ligand orbitals. [19] The reduction in the Coulomb parameters is related to an increase in hybridization and indicates a decrease in importance of the core-valence interaction. A strong reduction can occur when the excitonic final states in XAS are not completely pulled below the valence band continuum. Reductions of about $50 \%$ are necessary for shallow core-hole edges as the $M_{2,3}$ (3s) edges of transition metals $[20,21]$ and the $O_{4,5}(5 \mathrm{~d})$ edges of actinides [22].

\section{B. Crystal Field}

The spectral lineshape is generally strongly affected by solid-state effects. To lowest order, these effects can be included by an effective crystal field, $H_{\mathrm{CF}}$ in Eq. (1). This not only describes the point-charge crystal field, but can often also account for some of the effects of the hybridization of the central ion with the surrounding ligands. The effect of the crystal field is to lower the symmetry causing a splitting of the states that are obtained in spherical symmetry, i.e. by including only Coulomb and spin-orbit interactions. The spectral lineshape changes because of the energy splittings caused by the crystal field and due to the change in the symmetry of the ground state.

Many conventions exist for parametrizing the effect of the ligand environment. In our code, for the crystal field we use a parametrization based on the point group of the ion in terms of Ballhausen or Wybourne parameters [23, 24]. For a shell with angular quantum number $l$, the 
crystal field is written in terms of spherical harmonics as

$$
H_{\mathrm{CF}}=\sum_{k, q} B_{k q} C_{q}^{k}
$$

with $0 \leq k \leq 2 l, k$ an even integer and $-k \leq q \leq k ; B_{k q}$ are the Wybourne crystal-field parameters.

The hermiticity of the Hamiltonian imposes $B_{k,-q}=$ $(-1)^{q} B_{k q}^{*}$. By separating the real and imaginary parts of the Wybourne parameters $B_{k q}=\operatorname{Re} B_{k q}+i \operatorname{Im} B_{k q}$, we can rewrite $H_{\mathrm{CF}}$ as

$$
\begin{aligned}
H_{\mathrm{CF}}=\sum_{k}\left\{B_{k 0} C_{0}^{k}+\right. & \\
\sum_{1 \leq q \leq k} & {\left[\operatorname{Re} B_{k q}\left(C_{q}^{k}+(-1)^{q} C_{-q}^{k}\right)\right.} \\
& \left.\left.+i \operatorname{Im} B_{k q}\left(C_{q}^{k}-(-1)^{q} C_{-q}^{k}\right)\right]\right\}
\end{aligned}
$$

The number of non-zero parameters, and algebraic relationships between them are determined by the point group symmetry. The Wybourne parameters $B_{k q}$ can be easily related to the Stevens parameters [25-27].

In the case of cubic octahedral symmetry $\left(O_{h}\right)$, the 4fold axis around $z$ limits the values of $q$ to $q=0, \pm 4$, and the invariance of the crystal field under 90-degree rotations about $x$ or $y$ forbids the term $B_{20}$, since the spherical harmonic $C_{0}^{2}$ is not preserved for those rotations, and relates the $q=0$ and $q=4$ parameters for the $k=4,6$ representations:

$$
\begin{aligned}
B_{44} & =\sqrt{\frac{5}{14}} B_{40} \\
B_{64} & =-\sqrt{\frac{7}{2}} B_{60}
\end{aligned}
$$

The only free parameters are $B_{40}$ and $B_{60}$. The crystal field Hamiltonian becomes

$$
\begin{aligned}
H_{\mathrm{CF}, O_{h}}= & B_{40}\left[C_{0}^{4}+\sqrt{\frac{5}{14}}\left(C_{4}^{4}+C_{-4}^{4}\right)\right] \\
& +B_{60}\left[C_{0}^{6}-\sqrt{\frac{7}{2}}\left(C_{4}^{6}+C_{-4}^{6}\right)\right]
\end{aligned}
$$

Another common notation in the cubic case is to use the parameters $V_{4}=B_{40} / 8$ and $V_{6}=B_{60} / 16$. [28] For an $f$ shell, the orbitals split into three independent representations: $a_{2 g}\left(f_{x y z}\right), t_{1 g}\left(f_{x^{3}}, f_{y^{3}}, f_{z^{3}}\right)$, and $t_{2 g}\left(f_{x\left(y^{2}-z^{2}\right)}\right.$, $\left.f_{y\left(x^{2}-z^{2}\right)}, f_{z\left(x^{2}-y^{2}\right)}\right)$ with energies

$$
\begin{aligned}
& \varepsilon_{a_{2 g}}=\frac{80}{143} B_{60}-\frac{4}{11} B_{40} \\
& \varepsilon_{t_{1 g}}=\frac{100}{429} B_{60}+\frac{2}{11} B_{40} \\
& \varepsilon_{t_{2 g}}=-\frac{60}{143} B_{60}-\frac{2}{33} B_{40}
\end{aligned}
$$

TABLE I: Crystal-field splitting energies for the different irreducible representation $\Gamma$ with components $\gamma$ for $l=2$ in tetragonal symmetry $\left(D_{4 h}\right)$.

\begin{tabular}{lcc}
\hline$\Gamma$ & $\gamma$ & $\varepsilon_{\Gamma}$ \\
\hline$a_{1 g}$ & $3 z^{2}-r^{2}$ & $6 D q-2 D s-6 D t$ \\
$b_{1 g}$ & $x^{2}-y^{2}$ & $6 D q+2 D s-D t$ \\
$b_{2 g}$ & $x y$ & $-4 D q+2 D s-D t$ \\
$e_{g}$ & $y z, z x$ & $-4 D q-D s+4 D t$ \\
\hline
\end{tabular}

For a $d$-shell $(l=2)$ the term $B_{60}$ does not contribute, and the orbitals split between $e_{g}$ and $t_{2 g}$ orbitals at energies $\frac{2}{7} B_{40}$ and $-\frac{4}{21} B_{40}$ The parameter $B_{40}$ can be related to the commonly used $10 D q$ parameter for octahedral splitting by $10 D q=\frac{10}{21} B_{40}$. We can generalize the splitting in a $d$-shell to tetragonal symmetry and relate $B_{20}$, $B_{40}$ and $B_{44}$, to the parameters $D q, D s$ and $D t[1,29]$,

$$
\begin{aligned}
B_{20} & =-7 D s \\
B_{40} & =21(D q-D t) \\
B_{44} & =21 \sqrt{\frac{5}{14}} D q
\end{aligned}
$$

The splitting of the valence shell orbitals is determined by the point-symmetry group of the crystalline environment. By making a unitary transformation to a symmetry-adapted basis it is always possible to write the crystal-field Hamiltonian as a sum over irreducible representations,

$$
H_{\mathrm{CF}}=\sum_{\Gamma \gamma} \varepsilon(\Gamma) c_{\gamma}^{\dagger} c_{\gamma}
$$

where $\varepsilon_{\Gamma}$ is the energy of the $\Gamma$ representation of the point group, and $c_{\gamma}^{\dagger}$ is the creation operator for an electron in the $\gamma$ orbital belonging to the $\Gamma$ representation. In the case of cctahedral Symmetry $\left(O_{h}\right)$ for a $d$-shell, the $e_{g}\left(d_{3 z^{2}-r^{2}}, d_{x^{2}-y^{2}}\right)$ and $t_{2 g}\left(d_{x y}, d_{y z}, d_{x z}\right)$ orbitals are separated by an energy $10 D q$. For tetragonal symmetry $\left(D_{4 h}\right)$ the crystal-field splittings of a $d$-shell orbitals are usually given in terms of the parameters $D q, D s$ and Dt (see table I).

\section{Hybridization}

In many cases, the inclusion of an effective crystal field can lead to a satisfactory interpretation of the spectral line shape, in particular for x-ray absorption spectroscopy. However, when one is dealing with stronglycovalent materials or when interpreting x-ray photoemission, such an approach is inadequate and the ligands need to be included explicitly. The final term $H_{\text {hybridization }}$ in the Hamiltonian in Eq. (1) describes the hybridization of the central ion with the surrounding ligands. Although the number of ligand orbitals is large, the ion 
only hydridizes with particular symmetry combinations of orbitals. For example, a $d$ orbital only hybridizes with five linear combinations of ligand orbitals that have the same symmetry properties as the $d$ orbitals. This can be included in the model by an additional shell of effective ligands and take into account configurations $d^{n}$, $d^{n+1} \underline{L}^{1}, d^{n+2} \underline{L}^{2} \ldots$ where $\underline{L}^{n}$ denotes $n$ holes in the ligand shell. Including additional configurations in the model increases the computational cost of constructing and diagonalizing our Hamiltonian because of the increase in the size of the Hilbert space. We include in the Hamiltonian a hybridization term that mixes the valence orbitals with an effective ligand shell $L$ with the same number of orbitals as the valence shell. We consider only the linear combinations of orbitals for a particular point symmetry group that couple to the valence shell (i.e. they belong to the same irreducible representation as the valence shell orbitals)

The hybridization term is written as

$$
H_{\mathrm{hyb}}=\sum_{\Gamma \gamma} T_{d L}(\Gamma)\left(d_{\gamma}^{\dagger} L_{\gamma}+L_{\gamma}^{\dagger} d_{\gamma}\right)+\sum_{\Gamma \gamma} \varepsilon_{L}(\Gamma) L_{\gamma}^{\dagger} L_{\gamma}
$$

$\varepsilon_{L}(\Gamma)$ is the on-site energy for the electrons in the ligand shell depending on the irreducible representation $\Gamma$ to which the ligand orbital $\gamma$ belongs to. This displacement is produced by the hybridization between the valence orbitals. $d_{\gamma}^{\dagger}$ and $L_{\gamma}^{\dagger}$ are the creation operators of an electron in the $d$ and ligand shells. The transfer integrals $T_{d L}(\Gamma)$ are written in terms of the Slater-Koster parameters [30]: $(p d \sigma),(p d \pi)$, related to the overlap between the $d$ and $p$ orbitals of the ligands. An additional term $T_{p p}=(p p \sigma)-(p p \pi)$ splits the $e_{g}$ and $t_{2 g}$ ligand effective orbitals $\left(T_{p p}\right.$ is approximately $\frac{1}{4}$ of the width of the ligand band).

The transfer integrals and ligand field splittings for octahedral 6-coordinated $\left(\mathrm{MO}_{6}\right)$ and planar 4-coordinated $\left(\mathrm{MO}_{4}\right)$ clusters, in the case of a $d$ metal-center and $p$ ligand orbitals [31] are shown in table II. When setting the values of the Slater-Koster parameters, $(p d \pi)$ is expected [32] to be slightly less than $(p d \sigma) / 2$. When considering changes in bond-length, we can use Harrison's relationship [33], i.e. that the $3 d-2 p$ and $2 p-2 p$ charge transfer integrals are proportional to the power -3.5 and -2 of the bond-distance, respectively.

The two parameters that determine the amount of covalent mixing between the valence shell of the metalcenter and the ligands are the transfer integrals $T$ in Eq. (13) and the charge transfer energy $\Delta$. We define $\Delta$ as the lowest cost in energy of removing one electron from the ligands and transferring it to the metal center, i.e., the difference between the lowest eigenenergies for the $d^{n}$ and $d^{n+1} \underline{L}$ configurations,

$$
\Delta=E\left(d^{n+1} \underline{L}\right)-E\left(d^{n}\right) .
$$

In the absence of hybridization, and neglecting the multiplet splitting, the total energy for a particular number
TABLE II: Hybridization parameters for the $\mathrm{TMO}_{6}$ and $\mathrm{TMO}_{4}$ clusters in the case of a $d$ metal-center and $p$ ligand orbitals for the group representations in $O_{h}$ and $D_{4 h}$ symmetry in terms of the Slater-Koster integrals $p d \sigma, p d \pi$.

\begin{tabular}{lll}
$\Gamma$ & $T(\Gamma)$ & $\varepsilon_{L}(\Gamma)$ \\
\hline $\mathrm{TMO}_{6}\left(O_{h}\right)$ & & \\
\hline$e_{g}$ & $\sqrt{3}(p d \sigma)$ & $\Delta+T_{p p}$ \\
$t_{2 g}$ & $-2(p d \pi)$ & $\Delta-T_{p p}$ \\
\hline $\mathrm{TMO}_{4}\left(D_{4 h}\right)$ & & \\
\hline$a_{1 g}$ & $(p d \sigma)$ & $\Delta+T_{p p}$ \\
$b_{1 g}$ & $\sqrt{3}(p d \sigma)$ & $\Delta-T_{p p}$ \\
$b_{2 g}$ & $-2(p d \pi)$ & $\Delta+T_{p p}$ \\
$e_{g}$ & $-\sqrt{2}(p d \pi)$ & $\Delta$
\end{tabular}

of electrons can be approximated by

$$
E\left(d^{n}\right) \cong E_{0}+n \varepsilon_{d}+\frac{n(n-1)}{2} U_{v v}
$$

where $U_{v v}$ is the monopolar part of the valence-valence Coulomb interaction. The charge transfer energy is then

$$
\Delta \cong \varepsilon_{d}+n U_{v v}-\varepsilon_{L}
$$

However, $\Delta$ can differ from this value by several electronvolts when considering the full multiplet hamiltonian. In the Xclaim code, the energy of the ligand-shell in Eq. 13 is given by the parameter $\varepsilon_{L}$ and not by $\Delta$. In order to use the charge-transfer energy $\Delta$ as a parameter, we need to calculate $\varepsilon_{L}$ for a given $\Delta$. To do so, first we calculate the ground state energies $E_{G S}\left(d^{n}\right)$ and $E_{G S}\left(d^{n+1}\right)$ for the $d^{n}$ and $d^{n+1}$ configurations of the metal center without taking into account the ligand shell. In the final calculation including ligands, the energy level of the ligand shell is given in terms of $\Delta$ as $\varepsilon_{L}=E_{G S}\left(d^{n}\right)-E_{G S}\left(d^{n+1}\right)+\Delta$.

\section{CALCULATION OF X-RAY SPECTRA}

For a one-photon process where the photon is absorbed, we can write the transition probability using Fermi's golden rule

$$
I(\omega)=\sum_{f}|\langle f|T| g\rangle|^{2} \delta\left(E_{f}-E_{g}-\hbar \omega\right) .
$$

$E_{g}$ and $E_{f}$ are the energies of the ground $|g\rangle$ and final states $|f\rangle$, respectively; $\hbar \omega$ is the x-ray energy and $T$ is a transition operator that connects the ground state to the final states. The particular form of $T$ depends on the x-ray process that we are considering. Eq. (17) can be reexpressed as a Green's function of the final state

$$
I(\omega)=-\frac{1}{\pi} \operatorname{Im}\left\langle g\left|T^{\dagger} \frac{1}{E_{g}+\hbar \omega-H_{f}+i \Gamma} T\right| g\right\rangle,
$$


where $\Gamma$ is the broadening due to the finite core-hole lifetime. In the calculation first the lowest-energy eigenstate $|g\rangle$ of the initial-state Hamiltonian is obtained and the Green's function of the final-state Hamiltonian is calculated by using a continued fraction expansion.

\section{A. X-ray Absorption Spectroscopy (XAS)}

In x-ray absorption a core electron is promoted to the valence shell by an x-ray photon. The transition operator in this case is $T(E 1)=\boldsymbol{\epsilon} \cdot \mathbf{r}$ for dipolar transitions and $T(E 2)=(\boldsymbol{\epsilon} \cdot \mathbf{r})(\boldsymbol{k} \cdot \mathbf{r})$ for quadrupolar transitions. $\boldsymbol{\epsilon}$ is the $\mathrm{x}$-ray polarization, $\mathbf{r}$ is the position operator and $\mathbf{k}$ is the propagation vector of the light. The transition operators for dipolar and quadrupiolar transitions can be rewritten as spherical tensors

$$
\begin{aligned}
& T(E 1)=r \sum_{q}(-)^{q} \epsilon_{-q}^{(1)} C_{q}^{1} \\
& T(E 2)=r^{2} \sum_{q}(-)^{q} \sqrt{\frac{2}{3}}[\epsilon k]_{-q}^{(2)} C_{q}^{2},
\end{aligned}
$$

where the tensor product is defined as $[\epsilon k]_{q}^{(2)}=$ $\sum \epsilon_{q^{\prime}}^{1} k_{q^{\prime \prime}}^{1}\left\langle 11 q^{\prime} q^{\prime \prime} \mid 2 q\right\rangle$

The matrix elements of the spherical harmonics $C_{q}^{k}$ are given in (7). The radial matrix elements $r$ are constant for a given edge. The transition operators for light with helicities $\lambda=+1$ and $\lambda=-1$ correspond to setting $q=$ \pm 1 in (19). Light with linear polarization along the $z$-axis corresponds to $q=0$. Setting the radial matrix elements $r$ to unity and taking the light propagating along the $z$ axis $\mathbf{k}=\mathbf{k}_{z}$ and linear polarization along the $x$ and $y$ axes the transition operators for dipole and quadrupole transitions become

$$
\begin{aligned}
T\left(E 1, e_{x}\right) & =\frac{1}{\sqrt{2}}\left(-C_{-1}^{(1)}+C_{1}^{(1)}\right) \\
T\left(E 1, e_{y}\right) & =\frac{i}{\sqrt{2}}\left(C_{-1}^{(1)}+C_{1}^{(1)}\right) \\
T\left(E 2, k_{z}, e_{x}\right) & =\frac{1}{\sqrt{6}}\left(-C_{-1}^{(2)}+C_{1}^{(2)}\right) \\
T\left(E 2, k_{z}, e_{y}\right) & =\frac{i}{\sqrt{6}}\left(C_{-1}^{(2)}+C_{1}^{(2)}\right) .
\end{aligned}
$$

The program calculates linear and circular dichroism subtracting the XAS for different polarizations . X-ray magnetic circular dichroism (XMCD) is defined as the difference between the spectra for the incoming light with helicities $\lambda=+1$ and $\lambda=-1$.

For XMCD, sum rules give a straightforward way to obtain the orbital and spin magnetic moment from the integrated values of the measured spectra [34, 35]. Similarly, it is possible to get the expectation value of the spin-orbit coupling $\left\langle\sum_{i} \mathbf{l}_{i} \cdot \mathbf{s}_{i}\right\rangle$ from the branching ratio of the isotropic spectrum [36-38]. The application of sum rules is not exempt from problems. The derivation of the sum rules with spin-dependent operators, such as the spin and the spin-orbit coupling, is based on the assumption that $j$ is a good quantum number at a particular spinorbit split edge. However, mixing of the edges occurs as a result of other interactions, in particular the Coulomb core-valence interaction [39-41]. For the spin sum rule, the presence of the magnetic dipolar term $\left\langle T_{z}\right\rangle$ further complicates the determination of the value of the spin $[42,43]$. In addition to the calculation of the spectrum, Xclaim also calculates the expectation values of the most relevant tensor in the ground state. For a successful fit of the spectrum, these expectation values can provide a good estimate of these quantities in the material. Furthermore, they can serve as an additional check on the $\mathrm{x}$-ray absorption sum rules.

\section{B. X-ray Photo-emission Spectroscopy (XPS)}

In X-ray Photo-emission Spectroscopy (XPS) the kinetic energy of an emited electron is measured at a constant incident energy of the x-rays. We can calculate the core-level XPS with the cluster model similarly to the $\mathrm{x}$-ray absorption by using the annihilation of an electron in the core shell as the transition operator. We can write the isotropic XPS as

$$
I^{\mathrm{XPS}}(\varepsilon)=-\frac{1}{\pi} \operatorname{Im} \sum_{m \sigma}\left\langle g\left|c_{m \sigma}^{\dagger} \frac{1}{E_{g}-\varepsilon-H_{f}+i \Gamma} c_{m \sigma}\right| g\right\rangle,
$$

where $c_{m \sigma}$ annihilates an electron with spin $\sigma$ in the $m$ orbital of the core shell and $\varepsilon$ is the energy difference between the photoelectron and the incident photon.

XPS is an ionizing proccess that produces large screening effects and charge transfer satellites appear accompanying the main peak of the spectra [44]. The spectral shape has a strong dependence on the magnitudes of the valence-valence and core-valence monopolar part of the Coulomb interactions $U_{v v}$ and $U_{c v}$. When only considering the charge-transfer energy and the monopole parts of the Coulomb interaction, the energies of the $\left|\underline{c} d^{n+1} \underline{L}^{1}\right\rangle$ and $\left|\underline{c} d^{n+2} \underline{L}^{2}\right\rangle$ configurations relative to $\left|\underline{c} d^{n}\right\rangle$ are $\Delta-\bar{U}_{c v}$ and $2 \Delta+U_{v v}-2 U_{c v}$, respectively. The spectroscopy final states that have a hole in the core shell and the Coulomb core-valence potential pull down configurations with increasing number of electrons in the valence shell. For a configuration with $n$ electrons in the valence shell, its energy is decreased by $n U_{c v}$. This effect usually produces a reordering of the final state configurations and $\left|\underline{c} d^{n+1} \underline{L}^{1}\right\rangle$ appears below $\left|\underline{c} d^{n}\right\rangle$. These two configurations are usually termed the well-screened and the poorly screened final states.

\section{Photoemission and inverse photoemission}

We can also calculate the electron-removal and electron-addition spectra, which can be observed in va- 
lence photoemission spectroscopy (PES) and inverse photoemission spectroscopy (IPES), respectively. [45]. The angular integrated PES spectrum, given in terms of the difference between the energies of the photoelectron and incident photon $\varepsilon$ is

$$
I^{P E S}=-\frac{1}{\pi} \operatorname{Im} \sum_{m \sigma}\left\langle g\left|d_{m \sigma}^{\dagger} \frac{1}{E_{g}-\varepsilon-H_{f}+i \Gamma} d_{m \sigma}\right| g\right\rangle,
$$

where $d_{m \sigma}$ annihilates an electron with spin $\sigma$ in the $m$ orbital of the valence shell.

The IPES spectrum, as a function of the difference between the incident electron and emitted photon $\varepsilon$ is calculated as

$$
I^{I P E S}=-\frac{1}{\pi} \operatorname{Im} \sum_{m \sigma}\left\langle g\left|d_{m \sigma} \frac{1}{E_{g}+\varepsilon-H_{f}+i \Gamma} d_{m \sigma}^{\dagger}\right| g\right\rangle,
$$

where $d_{m \sigma}^{\dagger}$ creates an electron with spin $\sigma$ in the $m$ orbital of the valence shell.

\section{GRAPHICAL INTERFACE}

When the program is started it displays a window (Fig. 1) with entries for the chemical element, ionization state and edge to be calculated, as well as different Hamiltonian parameters. Once the ion and edge are chosen, the initial and final state electronic configurations are automatically generated. The program shows below the reduction values for the Slater integrals for the Coulomb interactions within the valence shell, and between core and valence. The default reduction factor is 0.8 .

In addition to setting the reduction factors of the Coulomb interactions, it is also possible to edit the full set of Slater integrals and spin-orbit parameters for the initial and final states by clicking in the button HartreeFock values: Edit. This opens a window (Fig. 2) where the Hartree-Fock parameters are separated into two different blocks for the ground and final configurations. The Slater integrals $F^{k}, G^{k}$ and spin-orbit parameters are labelled in terms of the different core and valence shells. After clicking $O k$, the values of the parameters are saved. The values of the Slater integrals given in the window are renormalized by the reduction factors specified in the main window.

The program allows the components of the magnetic field in the $x, y, z$ directions to be specified. The two choices (exchange and magnetic field) mean that the field is acting on the spin moment $\mathbf{S}$ or on the total magnetic moment of the ion $\mu=\mu_{B}(\mathbf{L}+2 \mathbf{S})$. The exchange fields are given by setting $\mu_{B} H$ in units of $\mathrm{eV}\left(\mu_{B}=5.79\right.$. $\left.10^{-5} \mathrm{eV} \cdot \mathrm{T}^{-1}\right)$.

For setting the crystal field splitting, one can select from a list of different symmetries and parametrizations for the crystal field. The main window allows to the values of the octahedral $(10 D q)$ and tetragonal $(D q, D s$ and $D t$ ) crystal-field parameters. For other crystal-field parametrizations, the parameters are set with pop-up dialog boxes. One can specify the values of the energies of the different real $d$-orbitals, or in the case of a general point group, it is possible to set the crystal field in terms of Wybourne parameters $B_{k q}$ (Fig. 3). In the case of an $f$-valence shell the only way to set up the crystal field is to specify Wybourne parameters. The selection of spherical in the pull-down menu means that there is no crystal field term in the Hamiltonian.

The pop-up dialog box for setting the Wybourne parameters contains a pull-down list to set the point-group symmetry. For high symmetry point groups (cubic, tetragonal, hexagonal) the program automatically disables the input boxes for the parameters that arerequired to be zero by symmetry. In the case of cubic octahedral symmetry, the only free parameters are $B_{40}$ and $B_{60}$. and the program automatically calculates $B_{44}$ and $B_{64}$. Selecting point group symmetry any in the pull-down list means that there is no constraint in the Wybourne parameters and all input boxes are activated.

The last group of parameters are related to the hybridization (implemented for $O_{h}$ and $D_{4 h}$ symmetry). The first box is the maximum number of holes in the ligand shell, this is the number of different electronic configurations taken into account $\left(d^{n}, d^{n+1} \underline{L}, d^{n+2} \underline{L}^{2} \ldots\right)$. The rest of the input boxes set the numerical values for the different parameters involved in the hybridization Hamiltonian: charge-transfer energy $\Delta$, isotropic coulomb interaction $\left(F^{0}\right)$ for the valence shell and for the attractive potential between the core-hole and valence electrons $\left(F_{c v}^{0}\right)$, and the Slater-Koster parameters $((p d \sigma),(p d \pi)$ and the difference $(p p \pi)-(p p \sigma))$

From the parameters given, the program sets the Hamiltonian, and calculates expectation values of quantum operators in the ground state (energy, spin and orbital angular momentum and expected electronic occupations of the valence and ligand shells) and calculates the spectra.

The calculated spectra are shown in the output window (Fig. 4). For each of the polarizations calculated, results are placed in a separate tab in the plot window. In the case of dichroism, the difference (dichroism) and average spectra for two polarizations are shown. The multiplet model cannot account for the absolute positioning of the absorption edge energy, so the program positions the edge according to the values tabulated for the binding energies of the core-electrons in different elements [46]. The calculated spectrum is displayed as poles (vertical bars) and also convoluted with the input core-hole lifetime and experimental broadenings. There are input boxes on the plot for setting the values of the Lorentzian and Gaussian broadenings. For core-hole spin-edges, it is possible to set an energy-dependent Lorentzian broadening divided by an energy set by the user. This is to account for possible differences in core-hole lifetime broadening of the two spin-orbit split edges due to the presence of additional Koster-Kronig processes at the edge at higher energy. 


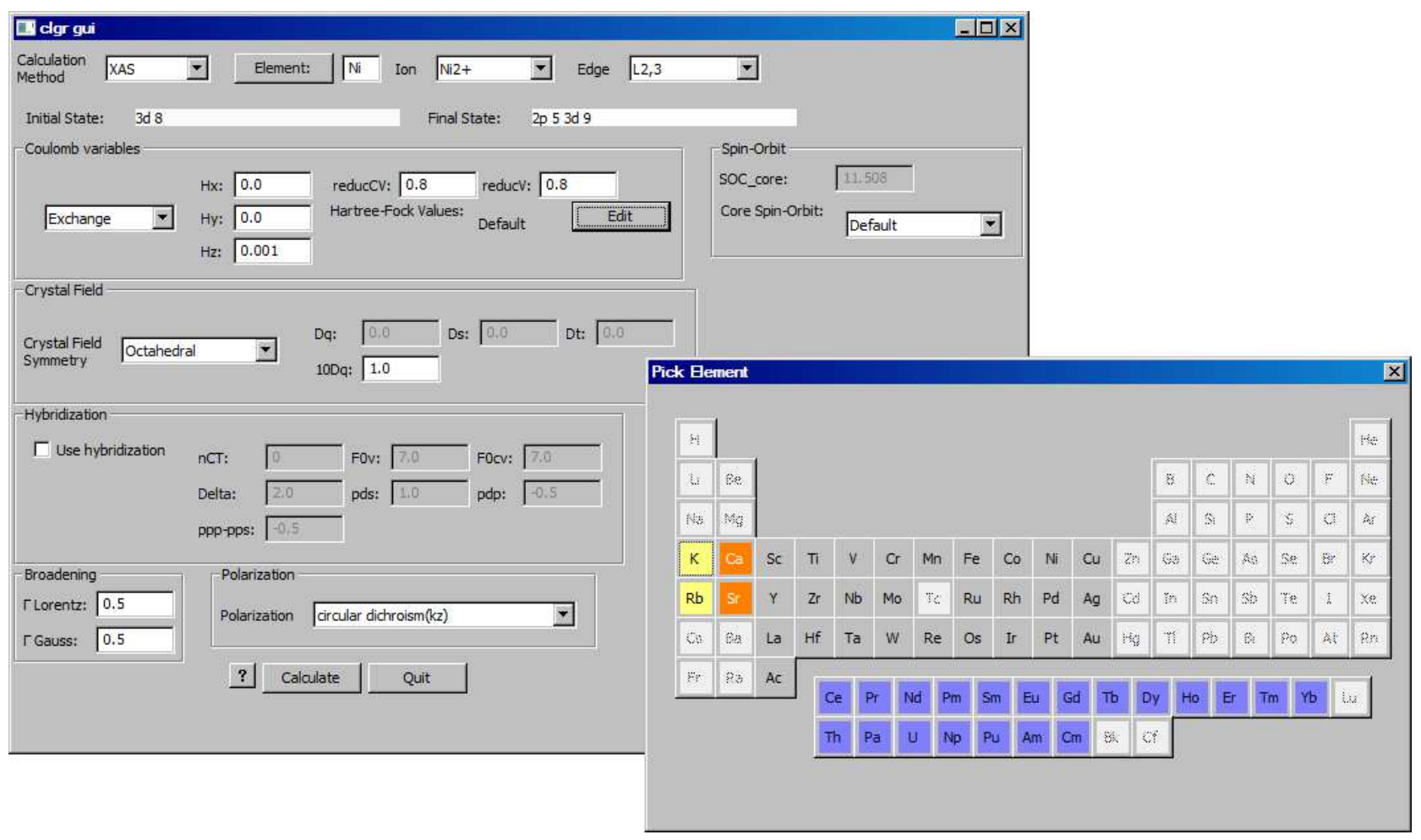

FIG. 1: Main input window and periodic table pop-up for the element selection. The main window contains the controls for setting up the main parameters for the spectra calculation divided into several areas: spectroscopy and ionization state, Coulomb and Spin orbit parameters, crystal field, hybridization, spectral broadenings and polarization.

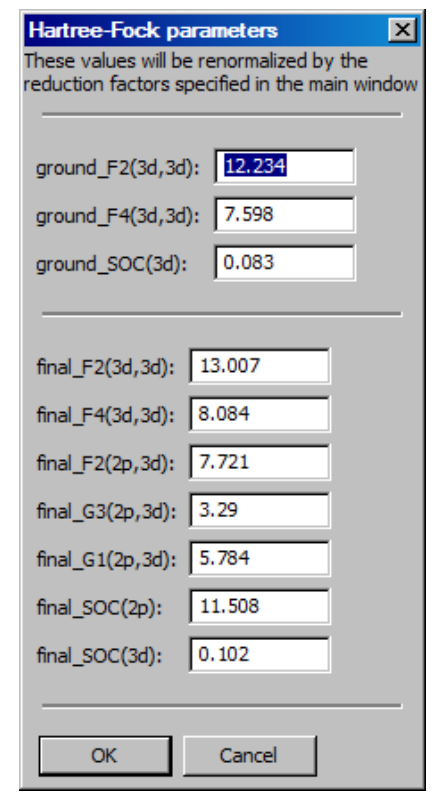

FIG. 2: Pop-up window showing the default Hartree-Fock parameters for the Slater integrals $F^{k}$ and $G^{k}$ and the spinorbit $\zeta$ of the core and valence shells. Customized values can be introduced in this window.

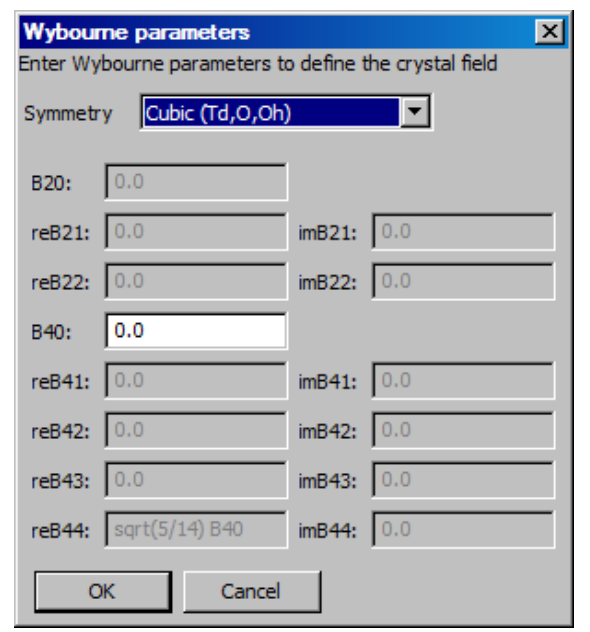

FIG. 3: Pop-up window for entering the crystal field when it is defined in terms of Wybourne parameters $B_{k q}$. The point group (cubic, tetragonal or hexagonal) can be selected from a pull-down list. The point symmetry places constraints between the parameters and only the input boxes for the independent parameters are active. The input boxes for the parameters that are zero or constrained are inactive. For the case of a general point group all the boxes are active and accept input. 


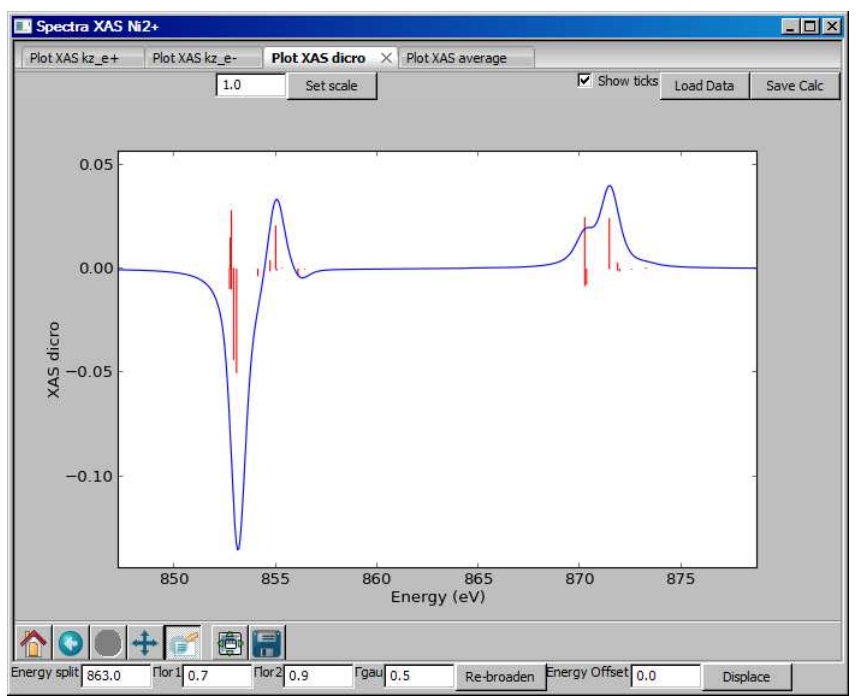

FIG. 4: Window displaying the calculated x-ray absorption spectra. Within the output window several tabs can be selected for viewing the absorption curve for each polarization (left and right), their difference (dichroism) and the average of the two polarizations. It possible to change the gaussian and lorentzian broadenings, set an arbitrary displacement in the $\mathrm{x}$-axis for the calculated spectra and to load experimental absorption curves for comparison with the calculated curve.

When the button Rebroaden is pressed, all polarization tabs in the window are recalculated. There are buttons on the plot window for loading experimental data to fit and to save the calculation results to a file.

Another window shows the parameters used for the calculation and the expectation values of different physical magnitudes in the ground state: number of holes in the ligand and valence shells, the components of the total spin $\mathbf{S}$ and orbital angular momentum $\mathbf{L}$ given in units of $\hbar$, spin-orbit coupling $\sum_{i} \mathbf{l}_{i} \cdot \mathbf{s}_{i}$, and the magnetic dipole operator $T_{z}$ that appears as an additional term in the XMCD spin sum rule [35] (see appendix A). For a $d$ valence shell the program also shows the individual occupation of the orbitals $d_{3 z^{2}-r^{2}}, d_{x^{2}-y^{2}}, d_{x y}, d_{y z}$, and $d_{z x}$.

\section{CONCLUSION}

We have presented a program for the calculation of core-hole spectroscopy from a multiplet model, which gives a good description of electron correlations and corevalence interaction. The conventions used for the model parameters are explained in terms of a general group theoretical treatment. The use of Wybourne parameters for the crystal field allows the treatment of any point symmetry and makes possible to fit x-ray spectra to a general crystal-field model. Although our code does not include a first principles calculation of crystal field parameters from the positioning of the ligands, the ab-initio crys- tal fields constructed by codes such as Hilbert $++[12]$ or MultiX [14] can be mapped into the Wybourne parameter set. Also, using this parametrizacion makes easy to relate the crystal-field obtained from x-ray spectra with the results derived by other techniques, such as inelastic neutron scattering. The treatment of charge transfer in Xclaim allows for valence-ligand charge transfer of an arbitrary number of electrons, while the codes derived from Thole and Butler programs $[9,10]$ are limited to one-electron charge transfer. The inclusion of several electrons charge transfer is important to accurately simulate satellite peaks in $\mathrm{x}$-ray photoemission spectroscopy (XPS). Applications include the fitting of x-ray spectra for the determination of crystal fields parameters and ground state configurations, sum rule error estimation or evaluating the effect in spectral shapes of charge transfer effects.

\section{ACKNOWLEDGEMENTS}

We are thankful to D. Haskel, U. staub, and J. A. Blanco for useful discussions. The periodic table was adapted from Robert Von Dreele's program pyFprime [47]. This work was supported by the U. S. Department of Energy (DOE), Office of Basic Energy Sciences, Division of Materials Sciences and Engineering under Award No. DE-FG02-03ER46097, the time-dependent $\mathrm{x}$-ray spectroscopy collaboration as part of the Computational Materials Science Network (CMSCN) under grants DE-FG02-08ER46540 and DE-SC0007091, and NIU Institute for Nanoscience, Engineering, and Technology. Work at Argonne National Laboratory was supported by the U. S. DOE, Office of Science, Office of Basic Energy Sciences, under contract No. DE-AC02-06CH11357.

\section{Appendix A: Coupled tensor operators}

In this appendix we define the coupled tensor operators, which are implemented in the program to calculate different quantum operators and physical magnitudes. For a shell with orbital $l$ and spin $s=\frac{1}{2}$ quantum numbers the unit tensor operator $w_{\zeta}^{x y z}$ is defined as [48, 49],

$$
\begin{aligned}
\mathrm{w}_{\xi \eta}^{x y}= & \sum_{m m^{\prime} \sigma \sigma^{\prime}}(-1)^{l-m^{\prime}} n_{l x}^{-1} n_{s y}^{-1}\left(\begin{array}{ccc}
l & x & l \\
-m^{\prime} & \xi & m
\end{array}\right) \\
& (-1)^{s-\sigma^{\prime}}\left(\begin{array}{ccc}
s & y & s \\
-\sigma^{\prime} & \eta & \sigma
\end{array}\right) c_{m^{\prime} \sigma^{\prime}}^{\dagger} c_{m \sigma}
\end{aligned}
$$

Where $a$ and $b$ are the unit tensor orbital and spin quantum numbers, with $-x \leq \xi \leq x,-y \leq \eta \leq y$. The normalization factor $n_{l x}$ is defined as

$$
n_{l x}=\left(\begin{array}{ccc}
l & x & l \\
-l & 0 & l
\end{array}\right)
$$


From the unit tensor operator we define the coupled tensor as,

$$
\mathrm{w}_{q}^{x y z}=\sum_{\xi \eta}(-1)^{x-\xi+y-\eta} n_{x y z}^{-1}\left(\begin{array}{ccc}
x & z & y \\
-\xi & \zeta & \eta
\end{array}\right) \mathrm{w}_{\xi,-\eta}^{x y}
$$

with $-z \leq \zeta \leq z . n_{x y z}$ is a normalization factor given by [50]

$$
n_{x y z}=\left(\begin{array}{ccc}
x & y & z \\
0 & 0 & 0
\end{array}\right)
$$

The double tensor operators $\mathrm{w}_{q}^{x y z}$ are used to get the ground state expectation values of physical observables: number of electrons in a shell $n_{h}=\mathrm{w}_{0}^{000}$, total spin $\mathbf{S}=$ $-s \mathbf{w}^{011}$ and orbital angular momenta $\mathbf{L}=-l \mathbf{w}^{101}$, spinorbit coupling $\sum_{i} \mathbf{l}_{i} \cdot \mathbf{s}_{i}=l s w_{0}^{110}$, and the magnetic dipole operator $\mathbf{T}=\sum_{i}\left(\mathbf{s}_{i}-3 \mathbf{r}_{i}\left(\mathbf{r}_{i} \cdot \mathbf{s}_{i}\right) / r_{i}^{2}\right)=-\frac{l}{2 l+3} \mathbf{w}_{0}^{211}$, which is relevant for the analysis of XMCD, where it appears as an additional term in the sum rule used to determine the spin angular momentum [35].
[1] C. J. Ballhausen, Introduction to Ligand Field Theory (1962).

[2] F. de Groot and A. Kotani, Core Level Spectroscopy of Solids (2008).

[3] G. van der Laan, Lecture Notes in Physics 697, 143 (2006).

[4] M. van Veenendaal, Theory of Inelastic Scattering and Absorption of X-rays (Cambridge University Press, Cambridge 2015).

[5] B. Thole, G. van der Laan, J. Fuggle, G. Sawatzky, R. Karnatak, and J.-M. Esteva, Phys. Rev. B 32, 5107 (1985).

[6] F. M. F. de Groot, J. C. Fuggle, B. T. Thole, and G. A. Sawatzky, Phys. Rev. B 42, 5459 (1990).

[7] A. Tanaka and T. Jo, J. Phys. Soc. Jpn. 63, 27882807 (1994).

[8] G. van der Laan and B. Thole, Phys. Rev. B 43, 13401 (1991).

[9] http://www.esrf.eu/computing/scientific/ MISSING/.

[10] E. Stavitski and F. M. de Groot, Micron 41, 687694 (2010).

[11] A. Mirone, S. S. Dhesi, and G. van der Laan, The European Physical Journal B 53, 23 (2006).

[12] A. Mirone, arXiv (2007), 0706.4170.

[13] A. Uldry, F. Vernay, and B. Delley, Phys. Rev. B 85, 125133 (2012).

[14] http://multiplets.web.psi.ch/manual.pdf.

[15] J. Fernandez-Rodriguez, B. Toby, and M. van Veenendaal, arXiv (2014), 1405.4313.

[16] https://subversion.xray.aps.anl.gov/xclaim/ xclaim.html.

[17] R. Cowan, The Theory of Atomic Structure and Spectra (1981).

[18] http://www.tcd.ie/Physics/People/Cormac. McGuinness/Cowan/.

[19] J. Zaanen, C. Westra, and G. Sawatzky, Physical Review B 33, 8060 (1986).

[20] R. Berlasso, C. Dallera, F. Borgatti, C. Vozzi, G. Sansone, S. Stagira, M. Nisoli, G. Ghiringhelli, P. Villoresi, L. Poletto, et al., Physical Review B 73, 115101 (2006).

[21] L. A. Wray, W. Yang, H. Eisaki, Z. Hussain, and Y.-D. Chuang, Phys. Rev. B 86, 195130 (2012).

[22] J. A. Bradley, S. Sen Gupta, G. T. Seidler, K. T. Moore, M. W. Haverkort, G. A. Sawatzky, S. D. Conradson, D. L. Clark, S. A. Kozimor, and K. S. Boland, Physical Review
B 81, 193104 (2010).

[23] J. Mulak and Z. Gajek, The effective crystal field potential (2000).

[24] M. W. Haverkort, Spin and orbital degrees of freedom in transition metal oxides and oxide thin films studied by soft $x$-ray absorption spectroscopy, Ph.D. Thesis (2005), URL http://kups.ub.uni-koeln.de/1455/.

[25] D. Newman and B. Ng, Crystal Field Handbook (2000).

[26] M. Rotter, M. D. Le, A. T. Boothroyd, and J. A. Blanco, Journal of Physics: Condensed Matter 24, 3201 (2012).

[27] Mcphase user manual, http://www2.cpfs.mpg.de/ $\sim$ rotter/homepage_mcphase/manual/manual.html.

[28] N. Magnani, G. Amoretti, S. Carretta, P. Santini, and R. Caciuffo, Journal of Physics and Chemistry of Solids 68, 2020 (2007).

[29] Z.-Y. Yang and Q. Wei, Physica B: Physics of Condensed Matter 370, 137 (2005).

[30] J. C. Slater and G. F. Koster, Physical Review 94, 1498 (1954).

[31] H. Eskes, L. H. Tjeng, and G. A. Sawatzky, Physical Review B 41, 288 (1990).

[32] L. F. Mattheiss, Physical Review B 5, 290 (1972).

[33] W. Harrison, Electronic Structure and the Properties of Solids (1980).

[34] B. T. Thole, P. Carra, F. Sette, and G. van der Laan, Phys. Rev. Lett. 68, 1943 (1992).

[35] P. Carra, B. T. Thole, M. Altarelli, and X. Wang, Physical Review Letters 70, 694 (1993).

[36] B. T. Thole and G. van der Laan, Physical Review A 38, 1943 (1988).

[37] B. T. Thole and G. van der Laan, Physical Review B 38, 3158 (1988).

[38] G. van der Laan and B. T. Thole, Physical Review Letters 60, 1977 (1988).

[39] J. P. Crocombette, B. T. Thole, and F. Jollet, Journal of Physics: Condensed Matter 8, 4095 (1996).

[40] G. van der Laan, K. Moore, J. Tobin, B. Chung, M. Wall, and A. Schwartz, Physical Review Letters 93, 097401 (2004).

[41] C. Piamonteze, P. Miedema, and F. de Groot, Physical Review B 80, 184410 (2009).

[42] J. Stöhr and H. König, Physical Review Letters 75, 3748 (1995).

[43] T. Oguchi and T. Shishidou, Physical Review B 70, 024412 (2004).

[44] G. van der Laan, C. Westra, C. Haas, and G. Sawatzky, 
Phys. Rev. B 23, 43694380 (1981).

[45] J. Zaanen and G. A. Sawatzky, Progress of Theoretical Physics Supplement, No. 101, pp. 231-270 101, 231 (1990).

[46] http://www.nist.gov/pml/data/ion_energy.cfm.

[47] https://subversion.xray.aps.anl.gov/trac/ pyFprime/.
[48] B. R. Judd, Second Quantization and Atomic Spectroscopy (1967).

[49] B. T. Thole, G. van der Laan, and M. Fabrizio, Physical Review B 50, 11466 (1994).

[50] M. van Veenendaal, European Journal of Physics 32, 947 (2011). 